\title{
Hardy-Littlewood maximal function on noncommutative Lorentz spaces
}

Jingjing Shao*

\author{
"Correspondence: \\ liangjingjing0868@126.com \\ College of Mathematics and System \\ Sciences, Xinjiang University, \\ Urumqi, 830046, China
}

\begin{abstract}
This paper is mainly devoted to the study of the Hardy-Littlewood maximal function on noncommutative Lorentz spaces and to obtaining $(p, q)$-(p, q)-type inequality for the Hardy-Littlewood maximal function on noncommutative Lorentz spaces.
\end{abstract}

MSC: 47A30; 47L05

Keywords: noncommutative Lorentz spaces; Hardy-Littlewood maximal function; von Neumann algebra

\section{Introduction}

In [1] Nelson defined the measure topology of $\tau$-measurable operators affiliated with a semi-finite von Neumann algebra. Fack and Kosaki [2] investigated generalized $s$-numbers of $\tau$-measurable operators and proved dominated convergence theorems for a gage and convexity (or concavity) inequality.

As for noncommutative maximal inequalities, a version of ergodic theory was given by Junge [3] and Junge, Xu [4]. In 2007, Mei [5] presented a version of noncommutative Hardy-Littlewood maximal inequality for an operator-valued function. In this paper, we study another version of Hardy-Littlewood maximal inequality introduced by Bekjan [6]. In [6], Bekjan defined the Hardy-Littlewood maximal function for $\tau$-measurable operators and, among other things, obtained weak $(1,1)$-type and $(p, p)$-type inequalities for the Hardy-Littlewood maximal function. In [6], for an operator $T$ affiliated with a semi-finite von Neumann algebra, the Hardy-Littlewood maximal function of $T$ is defined by

$$
M T(x)=\sup _{r>0} \frac{1}{\tau\left(E_{[x-r, x+r]}(|T|)\right)} \tau\left(|T| E_{[x-r, x+r]}(|T|)\right) .
$$

The classical Hardy-Littlewood maximal function of a Lebesgue measurable function $f$ : $\mathcal{R} \rightarrow \mathcal{R}$ denoted by $M f(x)$ is defined as

$$
M f(x)=\sup _{r>0} \frac{1}{m([x-r, x+r])} \int_{[x-r, x+r]}|f(t)| d t,
$$

where $m$ is a Lebesgue measure on $(-\infty,+\infty)(c f$. [7]). Moreover, a natural generalization of this is the case $f: \mathcal{R} \rightarrow \mathcal{R}$ and $\mu$, a Borel measure on $(-\infty,+\infty)$, where

$$
M_{\mu} f(x)=\sup _{r>0} \frac{1}{\mu([x-r, x+r])} \int_{[x-r, x+r]}|f(t)| d \mu(t) .
$$

(C) 2013 Shao; licensee Springer. This is an Open Access article distributed under the terms of the Creative Commons Attribution License (http://creativecommons.org/licenses/by/2.0), which permits unrestricted use, distribution, and reproduction in any medium, provided the original work is properly cited. 
As discussed by Bekjan in [6], let $\mu(A)=\tau\left(E_{A}(|T|)\right)$, where $A$ is a Borel subset of $(-\infty,+\infty)$. Then $\mu$ is a Borel measure and

$$
M T(x)=\sup _{r>0} \frac{1}{\mu([x-r, x+r])} \int_{[x-r, x+r]} t d \mu(t),
$$

i.e., $M T(x)$ is the Hardy-Littlewood maximal function $M_{\mu} f(x)$ of $f: \mathcal{R} \rightarrow \mathcal{R}$ defined by

$$
f(t)= \begin{cases}t, & t \in \sigma(|T|), \\ 0, & t \notin \sigma(|T|),\end{cases}
$$

with respect to $\mu$.

In view of spectral theory, $|T|$ is represented as

$$
|T|=\int_{\sigma(|T|)} t d E_{t}
$$

and $M T(|T|)$ is represented as $M T(x)$. Thus, for $T, M T(|T|)$ is considered as the operator analogue of the Hardy-Littlewood maximal function in the classical case. Therefore, roughly speaking, $M T(|T|)$ stands in relation to $T$ as $M f(x)$ stands in relation to $f$ in classical analysis.

In this paper, we study the Hardy-Littlewood maximal function on noncommutative Lorentz spaces. By primarily adapting the techniques in [8], we obtain the $(p, q)-(p, q)$ type inequality for the Hardy-Littlewood maximal function on noncommutative Lorentz spaces.

The remainder of this paper is organized as follows. Section 2 consists of some notations and preliminaries, including the noncommutative Lorentz spaces and their properties. In Section 3, we present the main result of this paper.

\section{Preliminaries}

Throughout the paper, let $\mathcal{M}$ be a finite von Neumann algebra acting on the Hilbert space $\mathcal{H}$ with a normal faithful tracial state $\tau$, and $C$ will be a numerical constant not necessarily the same in each instance. The identity in $\mathcal{M}$ is denoted by 1 , and we denote by $\mathcal{M}_{\text {proj }}$ the lattice of (orthogonal) projections in $\mathcal{M}$. A linear operator $T: \operatorname{dom}(T) \rightarrow \mathcal{H}$, with domain $\operatorname{dom}(T) \subseteq \mathcal{H}$, is said to be affiliated with $\mathcal{M}$ if $u T=T u$ for all unitary $u$ in the commu$\operatorname{tant} \mathcal{M}^{\prime}$ of $\mathcal{M}$. The closed densely defined linear operator $T$ affiliated with $\mathcal{M}$ is called $\tau$-measurable if for every $\epsilon>0$ there exists an orthogonal projection $P \in \mathcal{M}_{\text {proj }}$ such that $P(H) \subseteq \operatorname{dom}(T)$ and $\tau(1-P)<\epsilon$. The collection of all $\tau$-measurable operators is denoted by $\widetilde{\mathcal{M}}$. With the sum and product defined as the respective closures of the algebraic sum and product, $\widetilde{\mathcal{M}}$ is an $*$-algebra. For a positive self-adjoint operator $T$ affiliated with $\mathcal{M}$, we set

$$
\tau(T)=\sup _{n} \tau\left(\int_{0}^{n} \lambda d E_{\lambda}\right)=\int_{0}^{\infty} \lambda d \tau\left(E_{\lambda}\right)
$$

where $T=\int_{0}^{\infty} \lambda d E_{\lambda}$ is the spectral decomposition of $T$. 
Let $T$ be a $\tau$-measurable operator and $t>0$. The ' $t$ th singular number (or generalized $s$-number) of $T$ ' is defined by

$$
\mu_{t}(T)=\inf \left\{\|T E\|: E \in \mathcal{M}_{\text {proj }}, \tau(1-E) \leq t\right\} .
$$

By Proposition 2.2 of [2], we have

$$
\mu_{t}(T)=\inf \left\{s \geq 0: \lambda_{s}(T) \leq t\right\} \quad(t>0)
$$

where $\lambda_{s}(T)=\tau\left(E_{(s, \infty)}(|T|)\right)(s \geq 0)$ and $E_{(s, \infty)}(|T|)$ is the spectral projection of $|T|$ corresponding to the interval $(s, \infty)$. The reader is referred to [2] for basic properties and detailed information on generalized $s$-numbers and the distribution function of $\tau$-measurable operators.

Definition 2.1 (See, e.g., [9]) Let $T$ be a $\tau$-measurable operator affiliated with a finite von Neumann algebra $\mathcal{M}$, and let $0<p, q \leq \infty$. Define

$$
\|T\|_{L^{p, q}(\mathcal{M})}= \begin{cases}\left(\int_{0}^{\infty}\left(t^{\frac{1}{p}} \mu_{t}(T)\right)^{q} \frac{d t}{t}\right)^{\frac{1}{q}} & \text { if } q<\infty \\ \sup _{t>0} t^{\frac{1}{p}} \mu_{t}(T) & \text { if } q=\infty\end{cases}
$$

The set of all $T \in \widetilde{\mathcal{M}}$ with $\|T\|_{L^{p, q}(\mathcal{M})}<\infty$ is called the noncommutative Lorentz space, denoted by $L^{p, q}(\mathcal{M})$ with indices $p$ and $q$.

For convenience, we need the following Hardy inequalities in [10].

Lemma 2.2 If $q \geq 1, r>0$ and $f \geq 0$, then

$$
\left(\int_{0}^{\infty}\left[\int_{0}^{t} f(y) d y\right]^{q} t^{-r-1} d t\right)^{\frac{1}{q}} \leq \frac{q}{r}\left(\int_{0}^{\infty}[y f(y)]^{q} y^{-r-1}\right)^{\frac{1}{q}}
$$

and

$$
\left(\int_{0}^{\infty}\left[\int_{t}^{\infty} f(y) d y\right]^{q} t^{r-1} d t\right)^{\frac{1}{q}} \leq \frac{q}{r}\left(\int_{0}^{\infty}[y f(y)]^{q} y^{r-1}\right)^{\frac{1}{q}}
$$

Lemma 2.3 Let $0<r_{2}<p<\infty$ and $0<q, s<\infty$, then

$$
L^{p, q}(\mathcal{M}) \subset L^{r_{2}, s}(\mathcal{M})
$$

Let $L_{\mathrm{loc}}(\mathcal{M} ; \tau)$ be the set of all $\tau$-measurable operators such that

$$
\tau\left(|T| E_{I}(|T|)\right)<+\infty
$$

for all bounded intervals $I \subset[0,+\infty)$. 
Definition 2.4 (See, e.g., [6]) Let $T \in L_{\mathrm{loc}}(\mathcal{M} ; \tau)$, the maximal function of $T$ is defined by

$$
M T(x)=\sup _{r>0} \frac{1}{\tau\left(E_{[x-r, x+r]}(|T|)\right)} \tau\left(|T| E_{[x-r, x+r]}(|T|)\right)
$$

(let $\frac{0}{0}=0$ ). $M$ is called the Hardy-Littlewood maximal operator.

Remark 2.5 By the introduction of [6], we know that $M T(|T|)$ is represented as $M T(x)$. Hence, for $T \in L_{\mathrm{loc}}(\mathcal{M} ; \tau), M T(|T|)$ is considered as the operator analogue of the HardyLittlewood maximal function in the classical case. Therefore, roughly speaking, $M T(|T|)$ stands in relation to $T$ as $M f(x)$ stands in relation to $f$ in classical analysis. Also, in [6], it was proved that $M T(|T|)$ defined in Definition 2.4 was weak $(1,1)$-type and $(p, p)$-type. We refer the readers to [6] for more details and basic properties of $M T(|T|)$.

\section{Main result}

Lemma 3.1 Let $0<q<\infty, 1 \leq p, p_{0}, p_{1}<\infty$ and $p_{0} \neq p_{1}$ such that

$$
\frac{1}{p}=\frac{1-\theta}{p_{0}}+\frac{\theta}{p_{1}} \text { for some } 0<\theta<1
$$

Assume that $\mathcal{M}$ has no minimal projection, then there exists a constant $C$ such that $\forall T \in$ $L^{p, q}(\mathcal{M})$ we have

$$
\|M T\|_{p, q} \leq C\|T\|_{p, q} .
$$

Proof We assume that $p_{0}<p_{1}$. Theorem 2 of [6] and Lemma 2.3 imply that

$$
\|M T\|_{p_{0}, \infty} \leq\|M T\|_{p_{0}} \leq C\|T\|_{p_{0}} \leq C\|T\|_{p_{0}, m}
$$

and

$$
\|M T\|_{p_{1}, \infty} \leq\|M T\|_{p_{1}} \leq C\|T\|_{p_{1}} \leq C\|T\|_{p_{1}, m},
$$

where $m=\frac{1}{2} \min (1, q)$.

By Lemma 1.8 of [11], for all $t \in(0,1)$, we can take $P \in \mathcal{M}_{\text {proj }}$ such that $P|T|=|T| P$ and $\tau(P)=t$. Set $T_{1}=|T| P, T_{2}=|T|-T_{1}$, it is easy to check that $T_{1} \in L^{p_{0}, m}(\mathcal{M})$ and $T_{2} \in$ $L^{p_{1}, m}(\mathcal{M})$. Indeed, we see that $\mu_{v}\left(T_{1}\right)=\mu_{v}(|T| P)=\mu_{v}(T) \chi_{[0, t]}$ and $\mu_{v}\left(T_{2}\right)=\mu_{v}\left(|T| P^{\perp}\right)=$ $\mu_{v+t}(T)$. Thus we obtain

$$
\begin{aligned}
\left\|T_{1}\right\|_{p_{0}, m}^{m} & =\int_{0}^{\infty} v^{\frac{m}{p_{0}}-1} \mu_{\nu}(|T| P)^{m} d v=\int_{0}^{t} v^{\frac{m}{p_{0}}-1} \mu_{\nu}(T)^{m} d v \\
& =\int_{0}^{t}\left(v^{\frac{1}{p}} \mu_{v}(T)\right)^{m} v^{\frac{m}{p_{0}}-\frac{m}{p}-1} d v \\
& \leq\|T\|_{p, \infty}^{m} \int_{0}^{t} v^{\frac{m}{p_{0}}-\frac{m}{p}-1} d v \\
& \leq\left(\frac{q}{p}\right)^{\frac{m}{q}}\|T\|_{p, q}^{m} \frac{1}{\frac{m}{p_{0}}-\frac{m}{p}} t^{\frac{m}{p_{0}}-\frac{m}{p}}<\infty
\end{aligned}
$$


and

$$
\begin{aligned}
\left\|T_{2}\right\|_{p_{1}, m}^{m} & =\int_{0}^{\infty} v^{\frac{m}{p_{1}}-1} \mu_{v}\left(|T| P^{\perp}\right)^{m} d v=\int_{0}^{\infty} v^{\frac{m}{p_{1}}-1} \mu_{v+t}(T)^{m} d v \\
& \leq \int_{0}^{t} v^{\frac{m}{p_{1}}-1} \mu_{t}(T)^{m} d v+\int_{t}^{\infty} v^{\frac{m}{p_{1}}-1} \mu_{v}(T)^{m} d v \\
& \leq \frac{p_{1}}{m} t^{\frac{m}{p_{1}}} \mu_{t}(T)^{m}+\sup _{\nu>t}\left(v^{\frac{1}{p}} \mu_{v}(T)\right)^{m} \int_{t}^{\infty} v^{\frac{m}{p_{1}}-\frac{m}{p}-1} d v \\
& =\frac{p_{1}}{m} t^{\frac{m}{p_{1}}} \mu_{t}(T)^{m}+\|T\|_{p, \infty}^{m} \frac{1}{\frac{m}{p}-\frac{m}{p_{1}}} t^{\frac{m}{p_{1}}-\frac{m}{p}} \\
& \leq \frac{p_{1}}{m} t^{\frac{m}{p_{1}}-\frac{m}{p}}\left(\sup _{t>0} t^{\frac{1}{p}} \mu_{t}(T)\right)^{m}+\left(\frac{q}{p}\right)^{\frac{m}{q}} \frac{1}{\frac{m}{p}-\frac{m}{p_{1}}} t^{\frac{m}{p_{1}}-\frac{m}{p}}\|T\|_{p, q}^{m} \\
& =\left[\left(\frac{q}{p}\right)^{\frac{m}{q}} t^{\frac{m}{p_{1}}-\frac{m}{p}}\left(\frac{p_{1}}{m}+\frac{1}{\frac{m}{p}-\frac{m}{p_{1}}}\right)\right]\|T\|_{p, q}^{m}<\infty .
\end{aligned}
$$

Since

$$
\begin{aligned}
& \frac{1}{\tau\left(E_{[x-r, x+r]}(|T|)\right)} \tau\left(|T| E_{[x-r, x+r]}(|T|)\right) \\
& \leq \frac{1}{\tau\left(E_{[x-r, x+r]}(|T|)\right)} \tau\left(|T| P E_{[x-r, x+r]}(|T|)\right) \\
& \quad+\frac{1}{\tau\left(E_{[x-r, x+r]}(|T|)\right)} \tau\left(|T| P^{\perp} E_{[x-r, x+r]}(|T|)\right) \\
& =\frac{1}{\tau\left(E_{[x-r, x+r]}(|T|)\right)} \tau\left(\left|T_{1}\right| E_{[x-r, x+r]}\left(\left|T_{1}\right|\right)\right) \\
& \quad+\frac{1}{\tau\left(E_{[x-r, x+r]}(|T|)\right)} \tau\left(\left|T_{2}\right| E_{[x-r, x+r]}\left(\left|T_{2}\right|\right)\right) \\
& \leq \frac{1}{\tau\left(E_{[x-r, x+r]}\left(\left|T_{1}\right|\right)\right)} \tau\left(\left|T_{1}\right| E_{[x-r, x+r]}\left(\left|T_{1}\right|\right)\right) \\
& \quad+\frac{1}{\tau\left(E_{[x-r, x+r]}\left(\left|T_{2}\right|\right)\right)} \tau\left(\left|T_{2}\right| E_{[x-r, x+r]}\left(\left|T_{2}\right|\right)\right),
\end{aligned}
$$

taking supremum, we get

$$
M T(x) \leq M T_{1}(x)+M T_{2}(x),
$$

which implies that

$$
\|M T\|_{p, q} \leq C\left(\left\|M T_{1}\right\|_{p, q}+\left\|M T_{2}\right\|_{p, q}\right) .
$$

We estimate each term separately. For the first term, using (3.2) we get

$$
\begin{aligned}
\left\|M T_{1}\right\|_{p, q} & =\left\{\int_{0}^{\infty} t^{\frac{q}{p}}\left(\mu_{t}\left(M T_{1}\right)\right)^{q} \frac{d t}{t}\right\}^{\frac{1}{q}} \\
& =\left\{\int_{0}^{\infty} t^{\frac{q}{p}-\frac{q}{p_{0}}}\left(t^{\frac{1}{p_{0}}} \mu_{t}\left(M T_{1}\right)\right)^{q} \frac{d t}{t}\right\}^{\frac{1}{q}}
\end{aligned}
$$




$$
\begin{aligned}
& \leq C\left\{\int_{0}^{\infty} t^{q\left(\frac{1}{p}-\frac{1}{p_{0}}\right)}\left\|T_{1}\right\|_{p_{0}, m}^{q} \frac{d t}{t}\right\}^{\frac{1}{q}} \\
& =C\left\{\left[\int_{0}^{\infty} t^{-q\left(\frac{1}{p_{0}}-\frac{1}{p}\right)-1}\left(\int_{0}^{t} v^{\frac{m}{p_{0}}-1} \mu_{\nu}(T)^{m} d v\right)^{\frac{q}{m}} d t\right]^{\frac{m}{q}}\right\}^{\frac{1}{m}} .
\end{aligned}
$$

After replacing $r$ and $q$ respectively with $q\left(\frac{1}{p_{0}}-\frac{1}{p}\right)$ and $\frac{q}{m}$ in the first inequality in Lemma 2.2, we see that the last expression is estimated as follows:

$$
\begin{aligned}
& \leq \frac{C}{\left(\frac{m}{p_{0}}-\frac{m}{p_{1}}\right)^{\frac{1}{m}}}\left\{\int_{0}^{\infty}\left[v \cdot v^{\frac{m}{p_{0}}-1} \cdot \mu_{\nu}(T)^{m}\right]^{\frac{q}{m}} \cdot v^{-q\left(\frac{1}{p_{0}}-\frac{1}{p}\right)-1} d v\right\}^{\frac{1}{q}} \\
& =C\left(\int_{0}^{\infty} v^{\frac{q}{p}-1} \mu_{\nu}(T)^{q} d v\right)^{\frac{1}{q}} \\
& =C\|T\|_{p, q},
\end{aligned}
$$

i.e., $\left\|M T_{1}\right\|_{p, q} \leq C\|T\|_{p, q}$. To estimate the second term, by applying (3.3) we obtain

$$
\begin{aligned}
\left\|M T_{2}\right\|_{p, q}= & \left\{\int_{0}^{\infty} t^{\frac{q}{p}}\left(\mu_{t}\left(M T_{2}\right)\right)^{q} \frac{d t}{t}\right\}^{\frac{1}{q}} \\
= & \left\{\int_{0}^{\infty} t^{\frac{q}{p}-\frac{q}{p_{1}}}\left(t^{\frac{1}{p_{1}}} \mu_{t}\left(M T_{2}\right)\right)^{q} \frac{d t}{t}\right\}^{\frac{1}{q}} \\
\leq & C\left\{\int_{0}^{\infty} t^{q\left(\frac{1}{p}-\frac{1}{p_{1}}\right)}\left\|T_{2}\right\|_{p_{1}, m}^{q} \frac{d t}{t}\right\}^{\frac{1}{q}} \\
= & C\left\{\left[\int_{0}^{\infty} t^{q\left(\frac{1}{p}-\frac{1}{p_{1}}\right)-1}\left(\int_{0}^{\infty} v^{\frac{m}{p_{1}}-1} \mu_{v}\left(T_{2}\right)^{m} d v\right)^{\frac{q}{m}} d t\right]^{\frac{m}{q}}\right\}^{\frac{1}{m}} \\
\leq & C\left\{\int_{0}^{\infty} t^{q\left(\frac{1}{p}-\frac{1}{p_{1}}\right)-1}\left(\int_{0}^{t} v^{\frac{m}{p_{1}}-1} \mu_{t}(T)^{m} d v\right)^{\frac{q}{m}} d t\right\}^{\frac{1}{q}} \\
& +C\left\{\left[\int_{0}^{\infty} t^{q\left(\frac{1}{p^{2}}-\frac{1}{p_{1}}\right)-1}\left(\int_{t}^{\infty} v^{\frac{m}{p_{1}}-1} \mu_{v}(T)^{m} d v\right)^{\frac{q}{m}} d t\right]^{\frac{m}{q}}\right\}^{\frac{1}{m}} .
\end{aligned}
$$

For the second term $\left\{\left[\int_{0}^{\infty} t^{q\left(\frac{1}{p}-\frac{1}{p_{1}}\right)-1}\left(\int_{t}^{\infty} v^{\frac{m}{p_{1}}-1} \mu_{\nu}(T)^{m} d v\right)^{\frac{q}{m}} d t\right]^{\frac{m}{q}}\right\}^{\frac{1}{m}}$, replace $r$ and $q$ respectively with $q\left(\frac{1}{p}-\frac{1}{p_{1}}\right)$ and $\frac{q}{m}$ in the second inequality in Lemma 2.2, and we estimate the last expression as follows:

$$
\begin{aligned}
& \leq C\left\{\frac{p_{1}}{m} \mu_{t}(T)^{q} \int_{0}^{\infty} t^{\frac{q}{p}-1} d t\right\}^{\frac{1}{q}}+C\left\{\int_{0}^{\infty}\left[\nu \cdot v^{\frac{m}{p_{1}}-1} \cdot \mu_{\nu}(T)^{m}\right]^{\frac{q}{m}} v^{q\left(\frac{1}{p}-\frac{1}{p_{1}}\right)-1} d \nu\right\}^{\frac{1}{q}} \\
& =C\left\{\frac{p_{1}}{m} \mu_{t}(T)^{q} \int_{0}^{\infty} t^{\frac{q}{p}-1} d t\right\}^{\frac{1}{q}}+C\|T\|_{p, q} \\
& \leq C\|T\|_{p, q},
\end{aligned}
$$

i.e., $\left\|M T_{2}\right\|_{p, q} \leq C\|T\|_{p, q}$.

For the case of $p_{0}>p_{1}$, we may simply reverse the roles of $p_{0}$ and $p_{1}$ in the above proof. 
We have now shown that

$$
\|M T\|_{p, q} \leq C\|T\|_{p, q} .
$$

Theorem 3.2 Let $0<q<\infty, 1 \leq p, p_{0}, p_{1}<\infty$ and $p_{0} \neq p_{1}$ be such that

$$
\frac{1}{p}=\frac{1-\theta}{p_{0}}+\frac{\theta}{p_{1}} \quad \text { for some } 0<\theta<1 \text {. }
$$

Assume that $\mathcal{M}$ has minimal projections, then there exists a constant $C$ such that for all $T \in L^{p, q}(\mathcal{M})$ we have

$$
\|M T\|_{p, q} \leq C\|T\|_{p, q}
$$

Proof Since $\mathcal{M}$ has minimal projections, we consider the von Neumann algebra tensor product $\mathcal{M} \bar{\otimes} L^{\infty}([0,1] ; d m)$ denoted by $\overline{\mathcal{M}}$, equipped with the tensor product trace $\tau \otimes$ $d m$, where $d m$ is the Lebesgue measure on $[0,1]$, then $\overline{\mathcal{M}}$ has no minimal projection.

Let $|T|=\int_{\sigma(|T|)} \lambda d E_{\lambda}(|T|)$ be the spectral decomposition of $T$. Since

$$
\sigma(|T|)=\sigma(|T| \otimes 1)
$$

we have

$$
|T \otimes 1|=|T| \otimes 1=\int_{\sigma(|T|)} \lambda d\left(E_{\lambda}(|T|) \otimes 1\right)=\int_{\sigma(|T| \otimes 1)} \lambda d\left(E_{\lambda}(|T|) \otimes 1\right) .
$$

It is easy to check that $E_{\lambda}(|T|) \otimes 1$ is a spectral series for each $\lambda \geq 0$. Hence, for any interval

$$
I \subset \sigma(|T|)=\sigma(|T \otimes 1|)=\sigma(|T| \otimes 1),
$$

by the uniqueness of the spectral decomposition, we see that

$$
E_{I}(|T \otimes 1|)=E_{I}(|T|) \otimes 1
$$

For $\forall r>0$, since

$$
\tau\left(E_{[x-r, x+r]}(|T|)\right)=\int_{0}^{1} \tau\left(E_{[x-r, x+r]}(|T|)\right) d m=\tau \otimes d m\left(E_{[x-r, x+r]}(|T|) \otimes 1\right)
$$

and

$$
\begin{aligned}
\tau & \otimes d m\left(|T \otimes 1| E_{[x-r, x+r]}(|T|) \otimes 1\right) \\
& =\tau \otimes d m\left\{\left(|T \otimes 1| E_{[x-r, x+r]}(|T|) \otimes 1\right)^{*}\left(|T \otimes 1| E_{[x-r, x+r]}(|T|) \otimes 1\right)\right\}^{\frac{1}{2}} \\
& =\tau \otimes d m\left\{\left(E_{[x-r, x+r]}(|T|) \otimes 1\right)^{*}|T \otimes 1|\left(|T \otimes 1| E_{[x-r, x+r]}(|T|) \otimes 1\right)\right\}^{\frac{1}{2}} \\
& =\tau \otimes d m\left\{\left(E_{[x-r, x+r]}(|T|) \otimes 1\right)|T \otimes 1|^{2} E_{[x-r, x+r]}(|T|) \otimes 1\right\}^{\frac{1}{2}}
\end{aligned}
$$




$$
\begin{aligned}
& =\tau \otimes d m\left\{E_{[x-r, x+r]}(|T|) \otimes 1\left(|T|^{2} \otimes 1\right) E_{[x-r, x+r]}(|T|) \otimes 1\right\}^{\frac{1}{2}} \\
& =\tau \otimes d m\left\{\left(E_{[x-r, x+r]}(|T|) \otimes 1\right)(|T| \otimes 1)(|T| \otimes 1)\left(E_{[x-r, x+r]}(|T|) \otimes 1\right)\right\}^{\frac{1}{2}} \\
& =\tau \otimes d m\left(|T| E_{[x-r, x+r]}(|T|) \otimes 1\right) \\
& =\tau\left(|T| E_{[x-r, x+r]}(|T|)\right),
\end{aligned}
$$

which implies that

$$
M(T \otimes 1)(x)=M T(x)
$$

By an adaptation of the proof of Lemma 3.1, we deduce that

$$
\|M(T \otimes 1)(T \otimes 1)\|_{p, q} \leq C\|T \otimes 1\|_{p, q}
$$

With the trivial fact $\mu_{t}(T)=\mu_{t}(T \otimes 1)$, we know

$$
\|T \otimes 1\|_{p, q}=\|T\|_{p, q} .
$$

Combing this result with $M(T \otimes 1)(|T \otimes 1|)=M T(|T|)$, we infer that

$$
\|M T\|_{p, q} \leq C\|T\|_{p, q} .
$$

\section{Competing interests}

The author declares that she has no competing interests.

\section{Acknowledgements}

The author would like to thank the editor and anonymous referees for their helpful comments and suggestions on the quality improvement of the manuscript. This research is supported by the National Natural Science Foundation of China (No. 11071204) and XJUBSCX-2012002.

Received: 16 March 2013 Accepted: 2 August 2013 Published: 19 August 2013

\section{References}

1. Nelson, E: Notes on non-commutative integration. J. Funct. Anal. 15, 103-116 (1974)

2. Fack, T, Kosaki, H: Generalized s-numbers of $\tau$-measurable operators. Pac. J. Math. 123, 269-300 (1986)

3. Junge, M: Doob's inequality for non-commutative martingales. J. Reine Angew. Math. 549, 149-190 (2002)

4. Junge, $\mathrm{M}, \mathrm{Xu}$, Q: Noncommutative maximal ergodic theorems. J. Am. Math. Soc. 20(2), 385-439 (2007)

5. Mei, T: Operator valued Hardy spaces. Mem. Am. Math. Soc. 188, 881 (2007) (English summary)

6. Bekjan, TN: Hardy-Littlewood maximal function of $\tau$-measurable operators. J. Math. Anal. Appl. 322, 87-96 (2006)

7. Stein, E, Weiss, G: Introduction to Fourier Analysis on Euclidean Spaces. Princeton University Press, Princeton (1971)

8. Grafakos, L: Classical Fourier Analysis, 2nd edn. Springer, Berlin (2008)

9. Xu, Q: Noncommutative $L_{p}$-spaces and martingale inequalities. Book manuscript (2007)

10. Hunt, RA: On L(p, q) spaces. Enseign. Math. 12, 249-276 (1966)

11. Guido, D, Isola, T: Singular traces on semifinite von Neumann algebras. J. Funct. Anal. 134, 451-485 (1995)

doi:10.1186/1029-242X-2013-384

Cite this article as: Shao: Hardy-Littlewood maximal function on noncommutative Lorentz spaces. Journal of Inequalities and Applications 2013 2013:384. 